\title{
Criminologie
}

\section{L'opinion publique et les politiques criminelles}

\section{Yves Brillon}

Volume 19, numéro 1, 1986

Politiques et pratiques pénales. 25 ans de réflexion et d'action

URI : https://id.erudit.org/iderudit/017234ar

DOI : https://doi.org/10.7202/017234ar

Aller au sommaire du numéro

Éditeur(s)

Les Presses de l'Université de Montréal

ISSN

0316-0041 (imprimé)

1492-1367 (numérique)

Découvrir la revue

Citer cet article

Brillon, Y. (1986). L'opinion publique et les politiques criminelles. Criminologie, 19(1), 227-238. https://doi.org/10.7202/017234ar

\section{Résumé de l'article}

Public opinion plays a role in crime policies to the extent that judges take it into consideration in their sentences and the government authorities rely on its support, for example, when changing certain laws (criminalizing or decriminalizing behaviour that is or is no longer disapproved of by the community). However, public opinion is not always based on fact. For example, the public holds that the majority of criminals are dangerous, whereas violent crimes constitute only from $6 \%$ to $10 \%$ of all crimes. The population believes that the death sentence is an effective deterrent, whereas crimi-nological research has shown that this is not so. The "vox populi" necessitates caution. It speaks "emotionally", and although it must be heard, it is not always advisable to obey it. 


\section{L'OPINION PUBLIQUE \\ ET LES POLITIQUES CRIMINELLES \\ Yves Brillon*}

L'opinion publique est souvent une
force politique.

A. Sauvy

Public opinion plays a role in crime policies to the extent that judges take it into consideration in their sentences and the government authorities rely on its support, for example, when changing certain laws (criminalizing or decriminalizing behaviour that is or is no longer disapproved of by the community). However, public opinion is not always based on fact. For example, the public holds that the majority of criminals are dangerous, whereas violent crimes constitute only from $6 \%$ to $10 \%$ of all crimes. The population believes that the death sentence is an effective deterrent, whereas criminological research has shown that this is not so. The «vox populi» necessitates caution. It speaks «emotionally», and although it must be heard, it is not always advisable to obey it.

\section{CRISE DE LA JUSTICE ET... PEUR DU CRIME}

À l'heure actuelle, le système pénal, si souvent décrié, critiqué, blâmé, n'en demeure pas moins, pour beaucoup de citoyens, la seule institution capable de garantir à toute société un minimum d'ordre et de sécurité. Or, un peu partout dans le monde, les engrenages en cascade de la mécanique policière, judiciaire et correctionnelle paraîssent se gripper et fonctionner de façon de moins en moins efficace. L'opinion publique s'en émeut. Elle dénonce une montée vertigineuse de la violence. Ce qui a pour effet de susciter une augmentation considérable de la peur du crime au sein des populations. Cette peur s'avère d'autant plus redoutable qu'elle développe, chez les gens, une très grande méfiance les uns envers les autres. Le criminel n'a plus de visage. Il se perd dans la foule. Il voisine tout le monde. Il menace n'importe qui. Il est partout.

* Chercheur agrégé au Centre international de criminologie, Université de Montréal. 
Le rapport Figgie (1980) affirme que la peur des crimes de violence a rendu les Américains impuissants à les combattre. Peu à peu, aux États-Unis le crime se serait répandu, sournoisement, en se façonnant un visage de plus en plus menaçant, de plus en plus terrifiant; ... mais les gens s'y seraient résignés, incapables qu'ils sont d'entrevoir des mesures et des stratégies assez efficaces pour contrer le phénomène criminel. Ainsi, peu à peu, la criminalité et la probabilité d'en être victime auraient bouleversé le sentiment de sécurité et même, toujours selon les auteurs du Rapport Figgie, sapé le sens communautaire de la solidarité. De là à prétendre que la peur du crime est en train de briser ies liens sociaux, il n'y a qu'un pas. Et beaucoup de journalistes et de criminologues l'ont franchi allègrement en ajoutant que la peur du crime force les individus à s'isoler et à prendre en main leur propre protection. D'ailleurs, en cela, ils sont parfois directement encouragés par les agents pénaux qui démissionnent devant leurs propres responsabilités. C'est ce qui arriva quand les chefs de police de Los Angeles et de Miami dirent à leurs commettants : «Armez-vous, nous ne sommes plus en mesure de vous protéger» (Ward, 1982). Il n'y a rien là de très rassurant.

\section{LA PEUR... UN PRÉTEXTE À LA RÉPRESSION?}

À cause, en grande partie, de l'échec du système pénal à combattre la criminalité, un cercle vicieux s'est créé, bien défini par Conklin (1981). Cette spirale de la terreur peut se résumer de la façon suivante : la méfiance et les sentiments d'insécurité, dans une communauté, vont réduire le nombre de personnes dans les rues; ce qui aura une incidence directe sur le commerce, et indirecte sur le chômage et sur le développement économique de la région. Comme il y aura moins de gens dans les rues, celles-ci deviendront plus désertes et, par conséquent, plus dangereuses dans la mesure où le contrôle social informel du groupe s'étiolera. Moins il y aura de monde dans les rues, plus les gens qui s'y aventureront deviendront vulnérables. Et, de ce fait, la peur augmentera d'autant. Cette escalade de la peur semble devoir être sans limite... à moins de la faire changer de camp! C'est-à-dire de la transporter du côté des criminels.

En France, par exemple, Jacques Chirac déclarait, en octobre 1984 : «Le nombre des agressions s'est accru depuis 1981 dans des proportions insupportables... Il y va de la survie des libertés publiques, et donc de notre démocratie» (France-Soir Magazine, 
20-26 octobre 1984). Pour réinstaurer la paix sociale et le sentiment de sécurité parmi les citoyens, il paraît évident qu'il faille transformer la philosophie qui préside à l'application des lois pénales. Application qui, selon le maire de Paris, va, à l'évidence, dans le sens de l'indulgence. Ce qu'il faut modifier, en définitive, ce sont les politiques criminelles : "Il faut créer, en France, dit-il, une peine dissuasive et incompressible qui fasse changer la peur de camp et qui protège la société.» Accroissement des crimes de violence, impuissance des organismes de répression à traquer les malfaiteurs, échec des programmes de réhabilitation et de réinsertion sociale, voilà les principaux paramètres qui alimentent la crainte que développent les individus à l'égard de la criminalité.

\section{PEUR DU CRIME ET DISTORSION DE LA RÉALITÉ}

Cette crainte, par l'ampleur qu'elle peut prendre (souvent exagérée), exerce une pression sur les attorités publiques en vue de les inciter à instaurer des politiques criminelles plus dures, plus aptes à décourager les futurs délinquants. Comme les pratiques sont susceptibles d'évoluer plus rapidement que les lois, c'est au niveau des magistrats que l'opinion publique paraît d'abord ricocher. D'une recherche sur le sentencing, effectuée en France par Bernat de Celis auprès de 54 magistrats, il ressort que ceux-ci craignent que l'opinion publique les accuse de faiblesse, de sensiblerie et de laxisme s'ils recourent aux mesures de substitution à l'emprisonnement (tels les travaux communautaires et les joursamende). Ils redoutent d'être dévalorisés, tournés en ridicule, non seulement par la population mais par les délinquants eux-mêmes (cité par Pinatel, 1984). Les juges sont donc sensibles aux rumeurs et aux protestations des citoyens. Mais sans doute pas assez aux yeux de ces derniers qui estiment que, d'une façon générale, les tribunaux se montrent beaucoup trop indulgents dans les sentences qu'ils prononcent contre ceux qui sont reconnus coupables de crimes.

L'augmentation de la criminalité de violence et la trop grande clémence des prétoires forment deux réalités antinomiques qui ne peuvent qu'influer sur le sentiment d'insécurité de la population. Ces deux variables sont indissociables et ressortent comme telles des sondages d'opinion. Une recherche, faite par le G.R.A.C. ${ }^{1}$ (1984) indique que dans un échantillon canadien $(N=817 ; 1981)$ $72 \%$ des personnes estimaient que les sentences prononcées par les cours de justice n'étaient pas assez sévères. Un sondage Gallup, 
plus récent (1983), fait état de $79 \%$ des Canadiens qui trouvent que, d'une façon générale, les tribunaux sont trop complaisants envers les accusés. Dans leurs travaux, Doob et Roberts, tout comme l'avait fait le G.R.A.C.' pour la recherche "Attitudes» (1984), ont tenté de mettre en lumière l'image du criminel qui servait de référence, aux individus, pour évaluer la sévérité des sentences.

Les données des chercheurs ontariens indiquent que $45 \%$ des personnes interrogées pensent spontanément à des criminels dangereux ou à des récidivistes. Par ailleurs, $31 \%$ jugent que les tribunaux sont trop cléments envers tous les criminels. Or, il faut rappeler, ici, que ce même échantillon croit, dans une proportion de $74 \%$, qu'entre $30 \%$ à $100 \%$ des crimes commis au Canada impliquent de la violence c'est-à-dire, par exemple, que les victimes sont battues, sont violées à la pointe du révolver...; ou subissent d'autres sévices. Quant à la recherche du G.R.A.C., elle montre que, pour son échantillon canadien, $64 \%$ des interviewés se réfèrent à des criminels violents et endurcis alors que ceux-ci ne représentent qu'entre $6 \%$ à $10 \%$ de la totalité des délinquants. Dans la population canadienne, comme dans la plupart de celles de pays occidentaux, on surestime énormément le nombre de délinquants dangereux (au Canada, cette surestimation est d'au moins six fois trop élevée). Ce qui explique, dans une certaine proportion, les sentiments d'insécurité que manifestent les gens. L'insécurité reposerait donc, en grande partie, sur des connaissances fausses. De la même manière, les revendications des individus pour obtenir que les juges accordent des sentences plus sévères dériveraient d'une perception «distorsionnée» de la réalité criminelle. On peut illustrer ce fait en analysant quelques aspects de la crainte de victimisation.

\section{QUAND LA PRESSE «MÉDIATISE» LA PEUR}

"La grande peur». Ainsi titrait le magazine France-Soir, déjà cité, et dont les propos semblent d'avantage vouloir exacerber la

1. Cette recherche fut faite sur la demande du ministère du Solliciteur général du Canada qui la finança. L'échantillon «théorique» était constitué par une population fortement urbanisée (Montréal, Toronto et Winnipeg : $\mathrm{N}=614$ ) et par une population rurale des provinces de Québec, d'Ontario et du Manitoba $(N=203)$. L'échantillon total comportait 817 sujets. Les propos exprimés ici n'engagent que l'auteur. 
crainte des gens plutôt que de la conjurer. On ignore presque volontairement les éléments d'information susceptibles de ramener la violence à sa juste dimension et d'en définir les véritables contours victimologiques. Au contraire, on s'acharne, comme seule la bêtise peut le faire, à vouloir faire de la violence une hydre omnipotente dont chaque citoyen devient une victime potentielle :

Nul aujourd'hui ne se sent à l'abri. Nul ne peut dire : "Cela n'arrive qu'aux autres». Le malheureux client d'une banque pris en olage, ce peut être vous, ce peut être moi. Le passant malchanceux fauché par les éclats d'une bombe ce peut être un frère, ce peut être un ami. C'est ce sentiment de totale impuissance, l'impression d'être sans défense devant les formes les plus violentes de la criminalité qui habite aujourd'hui les Français (France-Soir, p. 3)

Et les chiffres à l'appui, on affirme péremptoirement que $65 \%$ des Français ne se sentent pas en sécurité. En fait, le sondage Louis Harris, auquel on se réfère, ne permet pas de lancer cette affirmation. La question posée était la suivante : «Vous personnellement, vous sentez-vous...»

- Tout à fait en sécurité? $35 \%$

- Moyennement en sécurité? $57 \%$

- pas du tout en sécurité?

$8 \%$

À la vue de ces chiffres, on peut tout aussi bien dire qu'il n'y a que $8 \%$ des Français qui ne se sentent pas du tout en sécurité et que $65 \%$ d'entre eux se sentent assez en sécurité. Le choix des mots, l'interprétation tendancieuse des chiffres font toute la différence et peut, auprès du public, ou apeurer ou rassurer. Au Canada, les statistiques de l'enquête du G.R.A.C. montrent que, en 1981, $90 \%$ des personnes interrogées avaient l'impression, dans leur quartier, d'être très $(30 \%)$ ou assez $(60 \%)$ en sécurité alors qu'il n'y en avait que $10 \%$ de l'échantillon qui se disaient peu $(7 \%)$ ou pas du tout $(3 \%)$ en sécurité.

\section{UNE FLAMBÉE DE VIOLENCE...}

\section{OU UNE IMAGE EN TROMPE-L'OEIL}

Mais ce sentiment d'être en sécurité, chez soi, dans son quartier, ne tévèle pas toute la vérité, rien que la vérité. Il y a sous cette apparente tranquillité d'esprit, une crainte diffuse qui empêche certaines personnes de sortir seules de chez elles ou de fréquenter certains lieux avoisinants. Il y a aussi, plus concrète- 
ment, la peur d'être victime. Et ce que les individus craignent le plus c'est d'être victimes d'actes de violence. Au Canada, si $90 \%$ de la population interrogée se sentent assez en sécurité là où ils habitent, on compte parmi cet échantillon $26 \%$ de gens qui évitent très souvent $(15 \%)$ ou souvent $(11 \%)$ de sortir scul le soir par peur du crime. À Montréal, le pourcentage de personnes qui craignent de s'aventurer seules à l'extérieur, à la tombée du jour, passe de $26 \%$ à $41 \%$ (dont $60 \%$ de femmes). Ce qui met en évidence la spécificité des grandes villes en ce qui a trait à la peur du crime. En milieu rural, cette peur ne touche que $13 \%$ des citoyens.

Et pour cause, puisque les grandes villes accaparent la part du lion en ce qui touche la criminalité. Aux États-Unis, en 1981, $80 \%$ de la totalité des meurtres, des viols et des vols qualifiés ont été commis dans des villes de 50000 habitants et plus. Or, ces villes ne représentent qu'environ $41 \%$ de la population. Il s'y commet donc deux fois plus de crimes qu'ailleurs. Les atteintes contre les biens sont un peu plus largement réparties dans la société américaine. Pour la même année, selon les chiffres fournis par Skogan (1983), environ $58 \%$ des cambriolages se sont produits dans les grands centres urbains. Par ailleurs, les enquêtes victimologiques et les statistiques de police révèlent que, aux États-Unis, les taux de criminalité violente sont en ville trois fois et demie plus élevés qu'en milieu rural. Il y a donc un parallélisme apparent entre la densité du phénomène criminel et la peur du crime. Toujours selon Skogan, une enquête nationale menée en 1982 a montré que $32 \%$ des habitants des régions rurales et des petites villes se déclaraient préoccupés par la criminalité. Cette proportion passait à $42 \%$ chez les habitants des zones suburbaines et à $71 \%$ chez ceux des grandes villes.

La recherche du G.R.A.C. montre, comme celles qui ont été faites aux États-Unis et en Europe, que si, dans l'ensemble, les personnes interrogées se disent relativement inquiètes devant l'augmentation de la criminalité au niveau national, il en va tout autrement au niveau du quartier. Ainsi la criminalité semble effrayer une majorité des gens interrogés, dans notre étude, mais cette préoccupation reste relativement générale et apparaît davantage comme une réaction sociale que comme une peur concrète découlant d'une menace perçue au niveau de la vie quotidienne. L'influence des médias n'est certainement pas étrangère à cette 
inquiétude grandissante que l'on peut constater parmi les Canadiens. Dans les années 70 , à peine un Canadien sur dix mentionnait la criminalité comme un problème social important (Courtis, 1970; CROP, 1972). Actuellement, selon nos données, une personne sur cinq $(19,3 \%)$ identifie la criminalité comme étant le problème le plus important au Canada. Parmi les trois problèmes jugés comme étant les plus graves, la criminalité est signalée au moins une fois sur deux $(55 \%)$, autant que la moralité et les valeurs $(54 \%)$; le problème prioritaire restant l'économie $(87 \%)$ (il s'agit, ici, des pourcentages cumulés sur les trois choix). Par ailleurs, à la question directe : «Est-ce que la criminalité vous préoccupe beaucoup, assez, un peu ou pas du tout?», $83,2 \%$ des répondants se sont dits beaucoup $(49,3 \%)$ ou assez $(33,9 \%)$ préoccupés par le phénomène criminel. Ces résultats montrent que, à l'heure actuelle, la criminalité laisse peu de gens indifférents.

Ce qui est lointain, étranger ou mal connu, inquiète beaucoup. Par contre, ce qui est familier et apprivoisé rassure davantage. Ainsi, les Canadiens, au niveau de leur localité, ne perçoivent pas de très grands changements dans l'évolution de la criminalité. On ne peut donc s'étonner que, dans la vie quotidienne, seule une minorité se sente personnellement menacée. Ainsi, à peine $10 \%$ des personnes interviewées ont le sentiment d'être en danger dans leur zone de résidence et, encore moins, $7,7 \%$ ont déjà songé à déménager parce qu'elles ne se sentaient pas en sécurité. Les gens qui perçoivent de hauts risques de victimisation forment, elles aussi, une minorité qui est loin d'être négligeable. Ils craignent davantage d'être victimes de vol $(38,4 \%)$ que d'une attaque contre la personne $(21,9 \%)$. Aux États-Unis, la crainte d'être victime de violence (c'est-à-dire de meurtre, d'assauts sexuels ou de viol, de blessures, de coups, d'une attaque à main armée) touche $41 \%$ de la population (The Figgie Report, 1980). Le taux de peur concrète à l'égard de la violence est donc de près de $20 \%$ plus élevé qu'au Canada.

\section{LA PEUR D'AVOIR PEUR...}

La peur est là. La plupart du temps sans commune mesure avec les risques encourus. Aux États-Unis, selon le Rapport Figgie, $17 \%$ des citoyens ont peur d'être assassinés alors que cette mort brutale ne frappe que $0,009 \%$ de la population; $55 \%$ des femmes 
craignent d'être violées; ce qui est une proportion 900 fois plus élevée que le pourcentage $(0,06 \%)$ de celles qui le sont; les gens qui appréhendent d'être l'objet d'un vol avec violence (23\%) sont 120 fois plus nombreux que les victimes de ce type de crimes $(0,19 \%)$. De plus, ce dont ont peur les gens c'est d'être attaqués, à l'extérieur de chez eux, par un inconnu. Or, comme le note Conklin $(1981$, p. 64$)$, les chances qu'a une personne d'être tuée par un inconnu sont au moins dix fois moins élevées que celles de mourir dans un accident de voiture. Ce que semble ignorer la population, c'est que beaucoup de crimes de violence sont commis par des gens qui se connaissent et qui, pour un certain nombre, sont apparentés.

Conklin cite une étude sur les meurtres à Philadelphie; étude qui a démontré que seulement $12 \%$ des affaires impliquaient des personnes qui étaient étrangères l'une par rapport à l'autre. Encore plus, dans $25 \%$ des meurtres la victime et l'aggresseur étaient de la même famille. Il se réfère aussi à une autre recherche, effectuée à Washington, D.C., qui est parvenue à la conclusion que seulement le tiers des victimes de viols et uniquement $19 \%$ des victimes d'assauts sexuels avaient été assaillis par des étrangers, par des inconnus. "Ainsi, conclut Conklin, un grand nombre de crimes, qui suscitent beaucoup de peur, sont commis par des personnes que les victimes connaissent déjà.» Pourquoi alors tant de crainte dans la population. Comment expliquer l'écart que l'on observe entre les risques objectifs d'être victime et la peur de la criminalité? Jusqu'à maintenant on n'a pas répondu de façon satisfaisante à cette interrogation fondamentale. Le manque de confiance dans le système pénal n'est sûrement pas étranger à l'inquiétude du public. Les politiques criminelles lui apparaissent, on l'a déjà mentionnés, faibles et inadéquates.

\section{LES POLITIQUES CRIMINELLES... OU L'INEFFICACITÉ DU SYSTĖME PÉNAL}

Les citoyens se rendent de plus en plus compte que le système de justice criminelle ne dispose pas de méthodes efficaces pour "contrôler» la criminalité, pour la domestiquer, pour la brider. Au contraire, pour la majorité des gens (G.R.A.C., 1984), il apparaît que la justice est inutilement compliquée et incompréhensible $(75 \%)$; qu'elle favorise les riches plus que les pauvres $(67 \%)$; qu'elle est inéquitable dans la mesure où un coupable 
peut toujours s'en sortir s'il dispose d'un bon avocat (74\%); que la lenteur des processus judiciaires est telle qu'elle incite les citoyens à ne pas déposer de plainte $(81 \%)$; que les lois actuelles sont en retard par rapport à la mentalité des gens et que les sentences que donnent les triburaux ne sont pas assez sévères $(72 \%)$. Anachronique, ésotérique, injuste, discriminatoire. embourbée, indolente, inhospitalière et trop indulgente, voilà les qualificatifs qui profilent l'image qu'ont de la justice la plupart des personnes interrogées. Et cette image se retrouve aux ÉtatsUnis (Figgie, 1980), en France (Peyrefitte, 1981) et dans la majorité des pays occidentaux.

L'impuissance des agences pénales à débusquer les malfaiteurs, à les identifier, à les confondre, à les arrêter et à les neutraliser suscite, dans la population, méfiance et crainte. Méfiance face à des institutions qui fonctionnent mal et qui s'avèrent inaptes à éliminer de la société des éléments indésirables. Crainte en ce qui a trait aux taux si faibles de résolution des affaires criminelles. Ce qui laisse croire qu'un grand nombre de malfaiteurs sont en liberté, puisqu'ils échappent à la vigilence des appareils étatiques de lutte contre le crime. Aux États-Unis, la plupart des chefs de police sont conscients, et ils le font savoir, que l'embauche d'un plus grand nombre de policiers n'est pas une solution au problème criminel. Ward (1982) rapporte qu'au Minnesota, et à travers le pays, une bonne part du blâme concernant l'accroissement de la criminalité est directement rejeté sur les tribunaux. Quand on a demandé aux gens, lors d'un sondage conduit par Newsweek, quel degré de confiance ils avaient dans la capacité des tribunaux de reconnaître la culpabilité d'un inculpé et de lui donner une sentence, $59 \%$ des personnes interrogées ont répondu : pas beaucoup, et $11 \%$ pas du tout.

D'après les données accumulées par Ward, les tribunaux sont fautifs, aux yeux du public, lorsqu'ils accordent la probation plutôt que l'emprisonnement; lorsqu'ils donnent de courtes sentences plutôt que de longues; lorsqu'ils rendent les peines «concurrentes» plutôt que consécutives; lorsqu'ils permettent que des vices de forme, lors des arrestations, et que des défauts de procédure, lors des audiences, débouchent sur la libération et l'acquittement des suspects; lorsqu'ils permettent aux accusés d'être libérés sous caution pendant leur procès. Ce sont là, sans parler du «plea bargaining», des comportements qui restent in- 
compris et suspects pour beaucoup de citoyens. Et cette incompréhension jette un discrédit sur les tribunaux. Parmi notre échantillon canadien, il y a $57 \%$ des répondants qui pensent que "les décisions des juges empêchent la police d'être efficace». Mais, il est important de le souligner de nouveau ici, que lorsque les gens blâment les tribunaux pour leur laxisme c'est parce qu'ils ont en tête l'image du criminel dangereux et celle de la criminalité de violence. C'est là une donnée capitale qu'il faut garder en vue en interprétant les attitudes du public envers le phénomène criminel et le système pénal.

\section{LES POLITIQUES CRIMINELLES... SELON LA POPULATION}

Dans le public, on exige des peines plus sévères envers les criminels. Toutefois, cette punitivité est exclusivement orientée vers les criminels dangereux. Dans notre recherche, celle du G.R.A.C., il n'y a que $5,4 \%$ des répondants qui exigeraient une peine de prison pour quelqu'un qui aurait commis un petit vol. Parmi les gens, $86 \%$ favoriseraient, en général dans un tel cas, une sanction de travaux communautaires. Même indulgence pour un criminel qui aurait commis plusieurs petits vols sans violence. Si $20 \%$ seraient enclins à recourir à l'emprisonnement, $80 \%$ ne verraient pas d'objection à ce que la sentence soit plutôt consacrée à effectuer des travaux utiles à la communauté. Toutefois, dès qu'il y a violence, on change de registre, on franchit un seuil d'intolérance. Même pour une personne qui commettrait, pour la première fois, un vol à main armée, $75 \%$ des personnes interrogées infligeraient spontanément une peine de prison. On rejoint, ici, les types de délits que Robert et Faugeron (1980) ont pu identifier, en France, au cours de leurs recherches qualitatives et quantitatives. Pour les gens, il y a le petit délit (peu grave et à faible intentionnalité) et le gros délit (grave et prémédité). Il y a les crimes auxquels les gens peuvent s'identifier... et les autres : ceux qui appellent le rejet, la neutralisation, voire même la peine de mort.

En ce qui concerne les politiques criminelles, la population semble croire au traitement des délinquants primaires et se montre prête à leur accorder leur chance. Pour les autres, les vrais criminels, $54 \%$ des citoyens les jugent irrécupérables. Ils préconisent, pour eux, l'enfermement et, pour les cas les plus dangereux, les assassins, la peine de mort (sanction, appuyée par $83,5 \%$ de l'échantillon canadien et $76 \%$ de l'échantillon québécois). 


\section{CONCLUSION}

Les gens, pour se former une idée de la criminalité au Canada, s'appuient surtout sur une expérience relativement abstraite, basée sur les informations véhiculées par les médias et par le discours public. On ne peut s'étonner que l'image qu'ils se font alors du phénomène criminel soit une image d'une criminalité de violence (actes contre la personne) dont le volume, selon eux, augmente de façon dramatique. En revanche, les jugements qui sont faits sur la criminalité, au niveau local, celui du quartier, ne sont influencés que par les expériences concrètes basées soit sur certains indices de l'environnement (activités de la police, personnes ou situations suspectes, etc.), soit sur une expérience directe de victimisation. L'image de la criminalité est, ici, celle d'une criminalité plutôt dirigée contre les biens, la plus fréquente, et dont les risques apparaissent également comme beaucoup plus probables que les risques d'une atteinte contre la personne. Également, l'évolution et l'ampleur de cette criminalité est aussi perçue, sous cet angle de proximité, comme étant beaucoup moins dramatique.

Ces résultats ont une implication importante pour les politiques criminelles. En effet, ils indiquent que les réactions sociales d'intolérance et de punitivité se déterminent, principalement, par rapport à la criminalité de violence et, beaucoup moins, par rapport à d'autres actes criminels; cette image étant parfaitement dominante au niveau des perceptions sociales influencées surtout par les médias de masse. Par contre, la crainte de victimisation dépend plutôt des risques perçus pour sa propriété et ses biens et n'est, par conséquent, pas très liée ni à la punitivité ni à d'autres mesures de tolérance à l'égard des criminels (discrimination dans l'emploi, facilitation de réinsertion sociale, etc.). Il est donc important de retenir, lorsque l'on parle de la punitivité et de l'intolérance du public, que ces attitudes visent surtout une catégorie relativement restreinte d'actes criminels (contre la personne); catégorie qui reste mineure mais qui, en focalisant toute l'attention, polarise la plus forte proportion de réactions punitives.

\section{RÉFERENCES}

DOOB, A. N. and J.V. ROBERTS (1982), Crime: Some Views of the Canadian Public, University of Toronto.

CONKLIN, J. E. (1981), Criminology, Macmillan Publishing Co. Inc., New York. 
COURTIS, M. C. (1970), Attitude to Crime and the Police in Toronto, University of Toronto, Center of Criminology.

C.R.O.P. Inc. (1972), les Normes sociales, Rapport final, Montrél.

FIGGIE REPORT (The) (1980), America Afraid, The Figgie Report on Fear of Crime, Part 1: The General Public, A-T-O Inc., Ohio.

FRANCE-SOIR MAGAZINE 1984, no 153 du 20 au 26 octobre 1984, Paris. GROUPE DE RECHERCHE SUR LES ATTITUDES ENVERS LA CRIMINALITÉ (1984), Les attitudes du public canadien envers les politiques criminelles, Recherche faite par Y. Brillon, Ch. Louis Guérin et M.C. Lamarche, Cahier no 1, les Cahiers de recherches criminologiques, Centre international de criminologie comparée, Université de Montréal.

PEYREFITTE, A. (1981), les Chevaux du Lax Ladoga, Plon, Paris.

PINATEL, J. (1984), Peines de substitution et criminologie, in Revue internationale de criminologie et de police technique, no 4, vol. XXXVII.

ROBERT, Ph. et C. FAUGERON (1980), la lustice et son public, Les représentations sociales du système pénal, Collection Déviance et Société, Masson-Médecine et Hygiène, Paris-Genève.

SKOGAN, W. G. (1983), «La sécurité dans les grandes villes américaines», in Revue internationale de criminologie et de pol. tech., no 4, vol. XXXVI.

WARD, D. A. (1982), How Fear of Crime Changed the Daily Living Habits of Most Americans, University of Minnesota. 